\title{
Analysis of regulatory mechanism after ErbB4 gene mutation based on local modeling methodology
}

\author{
C.L. Chen and J.W. Zhao
}

The Key Laboratory of Biomedical Information Engineering of Ministry of Education, Institute of Biomedical Analytical Technology and Instrumentation, School of Life Science and Technology, Xi'an Jiaotong University, Xi'an, China

Corresponding author: J.W. Zhao

E-mail: junwuzhaoxj@126.com

Genet. Mol. Res. 15 (2): gmr. 15028647

Received March 23, 2016

Accepted April 11, 2016

Published May 13, 2016

DOI http://dx.doi.org/10.4238/gmr.15028647

\begin{abstract}
ErbB4 is an oncogene belonging to the epidermal growth factor receptor family and contributes to the occurrence and development of multiple cancers, such as gastric, breast, and colorectal cancers. Therefore, studies of the regulation of ErbB4 in cancerigenic pathway will advance molecular targeted therapy. Advanced bioinformatic analysis softwares, such as ExPASy, Predictprotei, QUARK, and I-TASSER, were used to analyze the regulatory mechanism after ErbB4 gene mutation in terms of amino acid sequence, primary, secondary, and tertiary structure of the protein and upstream-downstream receptor/ ligands. Mutation of the 19th and 113th amino acids at the carboxyl terminus of ErbB4 protein did not affect its biological nature, but its secondary structure changed and protein binding sites were near 2 mutational sites; moreover, after mutation introduction, additional binding sites were observed. Tertiary structure modeling indicated that local structure of ErbB4 was changed from an $\alpha$ helical conformation into a $\beta$ chain folding structure; the $\alpha$ helical conformation is the
\end{abstract}


functional site of protein, while active sites are typically near junctions between helical regions, thus the helical structures are easily destroyed and change into folding structures or other structures after stretching. Mutable sites of ErbB4 is exact binding sites where dimer formed with other epidermal growth factor family proteins; mutation enabled the ErbB4 receptor to bind to neuregulin 1 ligand without dimer formation, disrupting the signal transduction pathway and affecting ErbB4 function.

Key words: ErbB4; Gene mutation; Molecular modeling

\section{INTRODUCTION}

The cDNA of the ErbB4 gene codes for 1308 amino acids is $6 \mathrm{~kb}$ in length. ErbB4 contains a hydrophobic transmembrane region composed of 26 amino acids, extracellular ligand domain divided by the transmembrane region and a $\mathrm{C}$-terminal intracellular region. The intracellular region is divided into tyrosine kinase, including 276 amino acids, and a carboxyl end consisting of 282 amino acids (Deng and Zhang, 2002; Qiu et al., 2008). As a cell surface receptor, ErbB4 is important for the functions of the neuregulin (NRG) and epidermal growth factor (EGF) family proteins and is involved in genetic transcription, cell proliferation and differentiation, and apoptosis. The protein functions in the maintenance of the heart, central nervous system, intestinal tract, stomach, and mammary tissue. Wang (2015) found that activated ErbB4 was necessary for heart development and cardiac muscle cells; Almohazey and Frey (2015) and Takahashi et al. (2015) showed that ErbB4 gene mutations were closely linked with mental illness such as schizophrenia, bipolar disorder, and amyotrophic lateral sclerosis. Schumacher et al. (2015) suggested that ErbB4 receptor tyrosine kinase could cause lesions in the colon epithelium in inflammatory bowels. Canfield et al. (2015) suggested that ErbB4 drives the differentiation of mammary tissue and induces lactoprotein production and lactation. However, similarly to other EGF family members, ErbB4 was shown to be related to the formation, apoptosis, and metastasis of tumor cells and the generation of blood vessels in tumors.

High ErbB4 expression in tumor tissues such as gastric cancer, breast cancer, lung cancer, colon cancer, and melanoma, indicates a role for this protein in tumor occurrence and growth (Soung et al., 2006; Bublil and Yarden, 2007; Scarfò et al., 2016; Williams et al., 2015). Cullum et al. (2015) found that ErbB4 is an oncogene in melanoma and Kataoka et al. (1998) indicated that the expression of ErbB4 protein in gastric cancer tissues was higher than that in normal tissues. Therefore, the regulatory mechanism of ErbB4 should be determined to facilitate the diagnosis and treatment of cancer. Furthermore, the intracellular region has been shown to be important in studies of carcinostasis. The BH3-only protein acts as a 'sensor' of apoptosis stimulation and is regarded as a necessary effective factor for initiating apoptosis. Naresh et al. (2006) found that HER4 intracellular domain (4ICD) of ErbB4 is a BH3-only protein that can promote the apoptosis of breast cancer cells; Jones (2008) found that the notch intracellular domain of ErbB4 activates the growth of mammary tissue and improves the occurrence of breast cancer and apoptosis of breast cancer cells. However, the specific action mechanism by which the ErbB4 intracellular region involved in regulation remains unclear. After collection of a large number of gastric cancer tissues, mutations in the intracellular region of the ErbB4 gene were detected by polymerase chain reaction and genetic sequencing, and the protein expression of ErbB4 protein was determined through immunohistochemical methods. 
ErbB4 protein expression remains high after mutation of the 19th and 113th amino acids at the carboxyl end of the intracellular region. Following mutation, tumor tissues stopped growing and were gradually reduced, demonstrating that ErbB4 mutations change the protein structure to disrupt its function. Therefore, we examined the structure of ErbB4 protein; using bioinformatics tools, local modeling for ErbB4 was conducted to determine the structural changes before and after mutation. Changes in the ErbB4 functional pathway caused by such changes were examined to provide a foundation for studies of molecular target therapy for tumor treatment.

\section{MATERIAL AND METHODS}

The 282 amino acids at the carboxyl end of the intracellular region of ErBb4 are shown in the supplementary information. Labeled amino acids were mutated, including the 19th amino acid from arginine to glycine and the 113th from arginine to glutamine. Changes in physiological characteristics before and after mutation of the carboxyl end of the intracellular region of ErBb4 were analyzed using ExPASy (http://web.expasy.org/protparam/); secondary structure and biding sites were analyzed using Predictprotei (https://www.Predictprotein.org/). Short sequences of approximately 20 amino acids before and after mutation of the carboxyl end in the intracellular region of ErBb4 protein evaluated by local modeling using QUARK and I-TASSER software developed by Zhang at the University of Michigan (Zhang, 2008). A 3D model using I-TASSER was established through LOMETS multithreading and fragment assembly of iterative templates. QUARK is a computerized algorithm used for modeling analysis of protein folding and tertiary structure based on an $a b$ initio prediction method. Using only the amino acid sequence, we used this program to predict an accurate three-dimensional model (Zhang, 2008; Xu and Zhang, 2012). Compared to homology modeling methods such as Swiss-model (Roy et al., 2012), neither of these tools require excessive homology templates for the modeling process; therefore, they are applicable for modeling for small sections of protein without effective homology templates.

\section{RESULTS}

\section{Analysis of amino acid sequence before and after ErbB4 protein mutation}

Changes in the physiological characteristics after mutation of the carboxyl end of the intracellular region of ErBb4 were analyzed using ExPASy (Table 1). Changes in mutated ErbB4 compared to wild-type protein were not significant, showing the same half-life and liposoluble index.

Table 1. Characteristics of ErbB4 before and after mutation.
\begin{tabular}{l|l|l}
\hline Parameters & Before mutation & After mutation \\
\hline Molecular weight & $31,766.2$ & $31,639.0$ \\
\hline PI value & 5.61 & 5.35 \\
\hline Polarity & Number of negatively-charged residues (Asp+Glu): 36 & Number of negatively-charged residues (Asp+Glu): 36 \\
& Number of positively-charged residues (Arg+Lys): 30 & Number of positively-charged residues (Arg+Lys): 28 \\
\hline Number of atoms & 4386 & 4364 \\
\hline Coefficient of light extinction & 1.021 & 1.025 \\
\hline Estimation of half-life & $4.4 \mathrm{~h}$ (mammals) & $4.4 \mathrm{~h}$ (mammals) \\
\hline Instability index & 58.40 (instable) & 57.00 (instable) \\
\hline Liposoluble index & 56.42 & 56.42 \\
\hline
\end{tabular}




\section{Changes of the secondary structure and biding sites of ErbB4 protein before and after mutation}

The secondary structure and biding sites of ErbB4 were analyzed using the online software Predictprotei (Figure 1). Although only 2 sites of ErbB4 were mutated, the secondary structure showed large changes; specifically, protein biding sites were mainly located near the 19th and 113th amino acids and a larger number of biding sites were observed after mutation.

\section{Changes of molecular modeling of tertiary structure before and after mutation of ErbB4 protein}

In this study, QUARK and I-TASSER were used to model the intracellular region carboxyl end of ErbB4. The short amino acid sequence of approximately 10 amino acids near the mutated sites were regarded as modeling objects. The results are shown in Figures 2 and 3.

\section{DISCUSSION}

Bioinformatics software was used to analyze changes in the protein structure of the ErbB4 oncogene and changes in its function resulting from mutations at the 19th and 113th amino acids in the intracellular region of the carboxyl end of ErbB4. ExPASy were used to analyze the changes in physiological characteristics after mutation (Table 1). The results showed that a single amino acid mutation in the intracellular region carboxyl end of ErbB4 did not influence the physiological characteristics of this region. This may be because during protein processing, some key amino acids showed a large impact on the protein structure. Changes in the secondary structure and protein binding sites of ErbB4 were analyzed using Predictprotei online software (Figure 1).

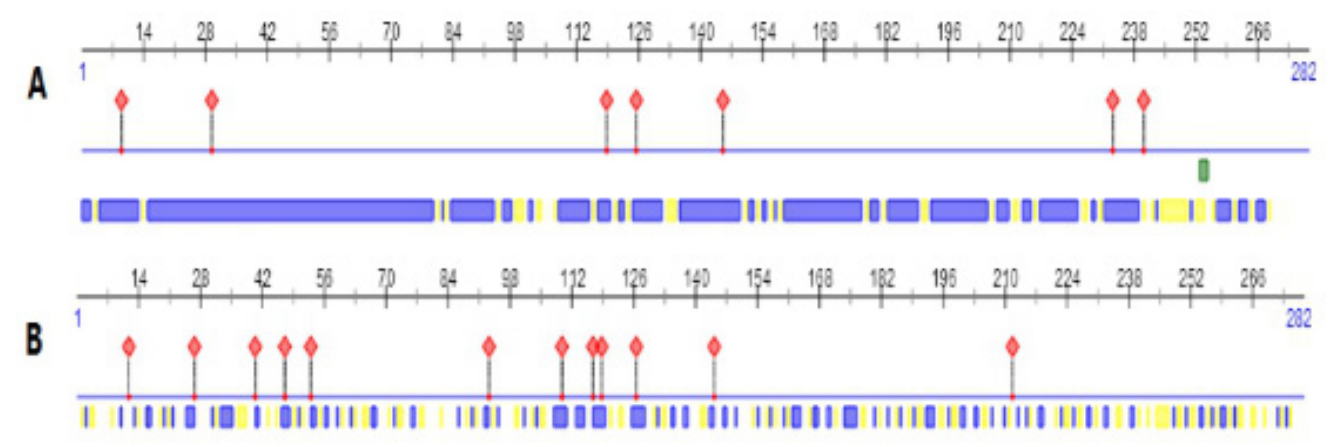

Figure 1. Changes in secondary structure and biding sites of ErbB4 protein. A. Represents the structure before mutation. B. Indicated structures after mutation, Rhombi in red color are protein-binding sites, Purple cylindricality represents secondary structure and solubility.

Only two sites of ErbB4 were changed, but large changes in the secondary structure were observed. Moreover, the protein biding sites were mainly near the 19th and 113th amino acids and the number of biding sites increased after mutation. This result indicates that these amino acids are key site for ErbB4 binding to its ligand. To clarify the structural changes 
in ErbB4 after its mutation, local modeling of the tertiary structure of ErbB4 before and after carboxyl end mutation was conducted using the QUARK and I-TASSER tools (Figures 2 and 3).

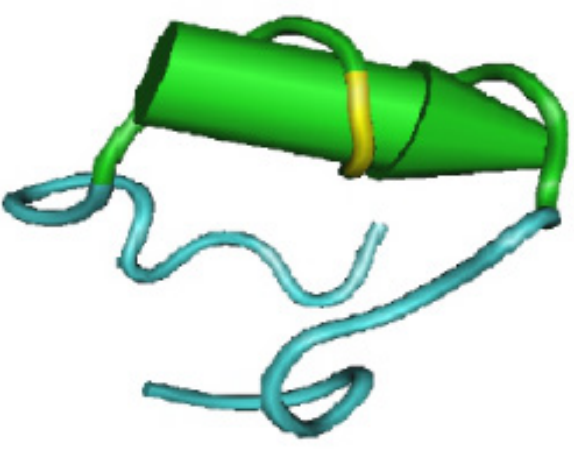

A

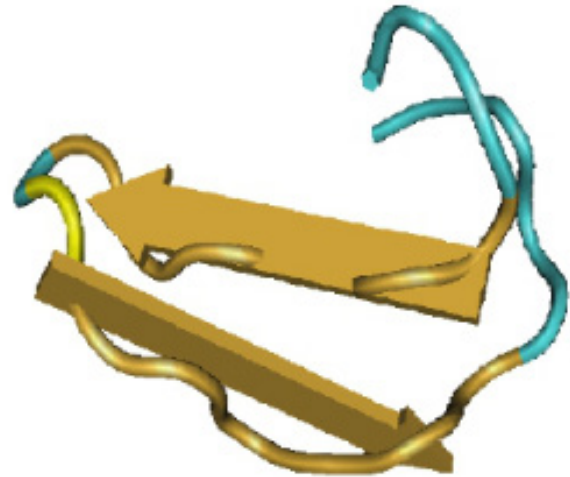

B

Figure 2. Structural characteristics before and after mutation of 19th mutational site.

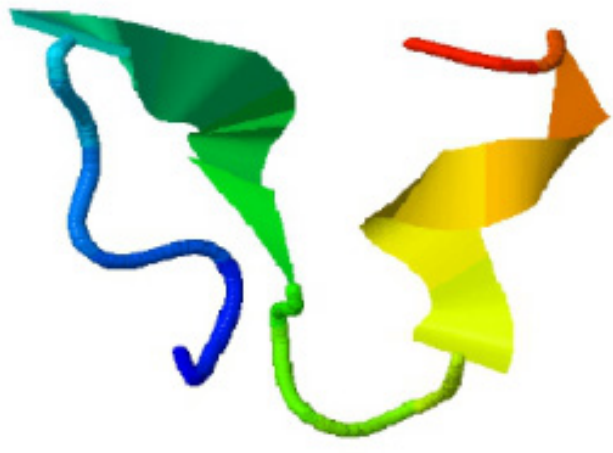

A

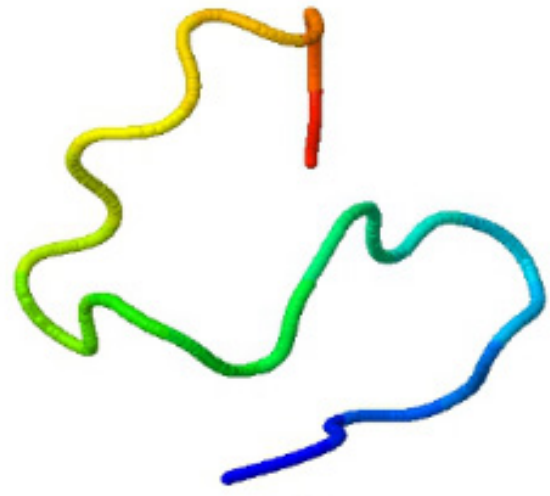

B

Figure 3. Structural characteristics before and after mutation of the 113th mutational site. A. Structure of the 113th mutation site before mutation (helical structure). B. Structure of the 113th mutation site after mutation (folding structure).

Mutation at the carboxyl end of ErbB4 transformed the $\alpha$ helical structure to a $\beta$ pleated sheet structure; additionally, the $\alpha$ helical structure is the functional part of protein and the active site are commonly located near the joints between the helical regions. Once the helical structure is stretched, it is easily broken and changes into a pleated sheet structure or other structures (Zhong, 2007). Therefore, changes in individual amino acid did not have a large influence on biological features because hydrogen bonds and Van der Waals forces, among others, form different structures during peptide processing; secondary structure analysis 
indicated that alterations in the binding site are important for ErbB4 binding to ligands, while changes in the tertiary structure further showed that the mutable part was the main functional region of ErbB4. Changes in the structure of ErbB4 affected the function of the protein in the cancerigenic pathway.

The ligand of ErbB4 protein is neurogenic differentiation factor, also known as neuregulin 1 (NRG1). Neurogenic differentiation can induce ErbB4 expression together with HER1 or HER2 to produce a dimer involved in the cancerigenic pathway of MAPK (Kainulainen et al., 2000; Xie et al., 2007); thus, only under the catalysis of neurogenic differentiation can ErbB4 form a dimer with ErbB1/2 to induce cell multiplication. If ErbB4 cannot form a dimer with HER1/2, it will act with the NRG1 ligand and STAT5a to induce cell differentiation. Gene mutations may limit dimer formation (Clark et al., 2005). Mutated sites of ErbB4 may form dimers with other EGF family proteins. Moreover, the mutations lead to binding between the ErbB4 receptor and the NRG1 ligand without producing dimers and interrupting the signal transduction pathway of ErbB4. The specific regulation mechanism pathway of ErbB4 protein before and after mutation in tumor tissues is shown in Figure 4.

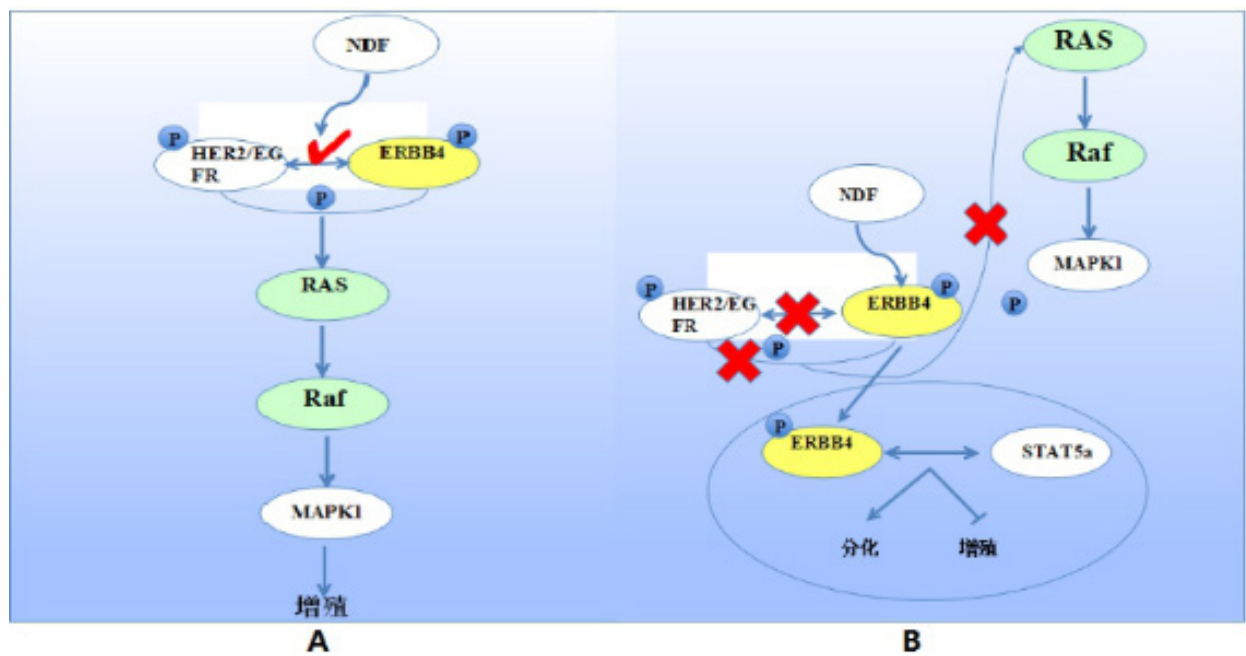

Figure 4. Functional pathways before and after mutation of ErbB4 protein. A. Represents the functional pathway before mutation of ErbB4 protein. B. Shows the functional pathway after mutation of ErbB4 protein.

Four members of the EGFR family are highly expressed in many tumor tissues and are closely related to the malignancy degree of the tumor, apoptosis, and transfer ability of invasion. Currently, iressa and herceptin are antineoplastic molecules targeting EGFR and ErbB2, respectively, and both have shown positive results for treating lung cancer and breast cancer (Ni and Zhang, 2015); therefore, the function of ErbB4 protein in the formation and development of tumors is gaining increasing attention. Ye et al. (2014) and Xu et al. (2008) found that miR-520a can regulate the expression of ErbB4. The multiplication and invasion of esophageal squamous carcinoma cells can be clearly restrained by interrupting the expression of ErbB4 protein; in a study by Starr et al. (2006), after introducing the HER4 gene into inexpressive lung cancer cell strains of H661 and H1299, the formation and growth 
of tumor cells were improved. An ErbB4 monoclonal antibody depressed the development of ErbB4-positive tumor cells. These findings show that the regulation mechanism of ErbB4 is significant for cancer treatment. Therefore, we evaluated the structural changes of ErbB4 by analyzing the mechanism and characteristics of ErbB4 to establish mathematical models for targeting of mutational sites and to analyze changes in ErbB4 function and specific regulation pathways resulting from mutation. This study lays a foundation for carrying out artificial mutation studies in tumor regions with high ErbB4 expression. Additionally, our results can be used in studies of molecular targeted therapy for treating tumors.

\section{Conflicts of interest}

The authors declare no conflict of interest.

\section{ACKNOWLEDGMENTS}

We thank all everyone who have contributed to this study, through advice and comments, particularly Dr. Zhao for technical expertise. This study was supported by funds \#132106000064 and \#152300410151.

\section{REFERENCES}

Almohazey D and Frey M (2015). The neuregulin receptors ErbB3 and ErbB4 have opposing effects on intestinal paneth cells. FASEB J. 29 (Suppl 1): 852.1.

Bublil EM and Yarden Y (2007). The EGF receptor family: spearheading a merger of signaling and therapeutics. Curr. Opin. Cell Biol. 19: 124-134. http://dx.doi.org/10.1016/j.ceb.2007.02.008

Canfield K, Li J, Wilkins OM, Morrison MM, et al. (2015). Receptor tyrosine kinase ERBB4 mediates acquired resistance to ERBB2 inhibitors in breast cancer cells. Cell Cycle 14: 648-655. http://dx.doi.org/10.4161/15384101.2014.994966

Clark DE, Williams CC, Duplessis TT, Moring KL, et al. (2005). ERBB4/HER4 potentiates STAT5A transcriptional activity by regulating novel STAT5A serine phosphorylation events. J. Biol. Chem. 280: 24175-24180. http://dx.doi. org/10.1074/jbc.M414044200

Cullum RL, Gupta RB and Riese DJ (2015). Abstract B17: A high-throughput screening process for the discovery of melanoma chemotherapeutics targeted at the ErbB4 receptor tyrosine kinase. Cancer Res. 75: B17.

Deng ZC and Zhang DH (2002). The expression of ErbB4/HER4 gene in tumors. Chin. J. Clini Oncol. 29: 680-682.

Jones FE (2008). HER4 intracellular domain (4ICD) activity in the developing mammary gland and breast cancer. $J$. Mammary Gland Biol. Neoplasia 13: 247-258. http://dx.doi.org/10.1007/s10911-008-9076-6

Kataoka H, Joh T, Kasugai K, Okayama N, et al. (1998). Expression of mRNA for heregulin and its receptor, ErbB3 and ErbB-4, in human upper gastrointestinal mucosa. Life Sci. 63: 553-564. http://dx.doi.org/10.1016/S0024$\underline{3205(98) 00306-3}$

Kainulainen V, Sundvall M, Määttä JA, Santiestevan E, et al. (2000). A natural ErbB4 isoform that does not activate phosphoinositide 3-kinase mediates proliferation but not survival or chemotaxis. J. Biol. Chem. 275: 8641-8649. http://dx.doi.org/10.1074/jbc.275.12.8641

Naresh A, Long W, Vidal GA, Wimley WC, et al. (2006). The ERBB4/HER4 intracellular domain 4ICD is a BH3-only protein promoting apoptosis of breast cancer cells. Cancer Res. 66: 6412-6420. http://dx.doi.org/10.1158/0008-5472. CAN-05-2368

Ni Y and Zhang S (2015). Erbb4 Signaling: an overlooked backup system? Cell Cycle 14: 1623. http://dx.doi.org/10.108 $\underline{0 / 15384101.2015 .1032653}$

Qiu C, Tarrant MK, Choi SH, Sathyamurthy A, et al. (2008). Mechanism of activation and inhibition of the HER4/ErbB4 kinase. Structure 16: 460-467. http://dx.doi.org/10.1016/j.str.2007.12.016

Roy A, Yang J and Zhang Y (2012). COFACTOR: an accurate comparative algorithm for structure-based protein function annotation. Nucleic Acids Res. 40: W471-W477. http://dx.doi.org/10.1093/nar/gks372

Scarfò I, Pellegrino E, Mereu E, Kwee I, et al.; European T-Cell Lymphoma Study Group (2016). Identification of a new 
subclass of ALK-negative ALCL expressing aberrant levels of ERBB4 transcripts. Blood 127: 221-232. http://dx.doi. org/10.1182/blood-2014-12-614503

Schumacher M, Bernard J, Punit S, Hsieh J, et al. (2015). Macrophage-specific ErbB4 is induced by DSS colitis and regulates macrophage survival. FASEB J. 29 (Suppl 1): 854.2.

Soung YH, Lee JW, Kim SY, Wang YP, et al. (2006). Somatic mutations of the ERBB4 kinase domain in human cancers. Int. J. Cancer 118: 1426-1429. http://dx.doi.org/10.1002/ijc.21507

Starr A, Greif J, Vexler A, Ashkenazy-Voghera M, et al. (2006). ErbB4 increases the proliferation potential of human lung cancer cells and its blockage can be used as a target for anti-cancer therapy. Int. J. Cancer 119: 269-274. http:// dx.doi.org/10.1002/ijc. 21818

Takahashi Yi, Shioya A, Matsumoto C, Sano T, et al. (2015). Immunohistochemical analysis of ErbB4, a causative gene product for ALS19, in the spinal cord of sporadic ALS patients (P4. 108). Neurology 84: (Suppl 14): P4. 108.

Wang Z (2015). Investigations into the role of the ErbB4 receptor in cardiomyocyte hypertrophy and adult cardiac function.

Williams CS, Bernard JK, Demory Beckler M, Almohazey D, et al. (2015). ERBB4 is over-expressed in human colon cancer and enhances cellular transformation. Carcinogenesis 36: 710-718. http://dx.doi.org/10.1093/carcin/bgv049

Xie F, Padival M and Siegel RE (2007). Association of PSD-95 with ErbB4 facilitates neuregulin signaling in cerebellar granule neurons in culture. J. Neurochem. 100: 62-72. http://dx.doi.org/10.1111/j.1471-4159.2006.04182.x

$\mathrm{Xu}$ D and Zhang Y (2012). Ab initio protein structure assembly using continuous structure fragments and optimized knowledge-based force field. Proteins 80: 1715-1735.

Xu S, Kitayama J, Yamashita H, Souma D, et al. (2008). Nuclear translocation of HER-4/c-erbB-4 is significantly correlated with prognosis of esophageal squamous cell carcinoma. J. Surg. Oncol. 97: 44-50. http://dx.doi. org $/ 10.1002 /$ jso.20892

Ye WG, Yao QL, Zhang MX, Huang HY, et al. (2014). miR-520 regulates erbb4 expression and suppression proliferation and invasion of esophageal squamous cell carcinoma. J. Southern Med. Univ. 2: 005.

Zhang Y (2008). I-TASSER server for protein 3D structure prediction. BMC Bioinformatics 9: 40. http://dx.doi. org/10.1186/1471-2105-9-40

Zhong SL (2007). Protein and helix. Mod. Med. Health 21: 804-805. 